\title{
Biomechanical evaluation and finite element analysis of axial-loading simulated experiment of wrist fracture
}

\section{Yuan-Wei Zhang}

Jiangxi Provincial People's Hospital Affiliated to Nanchang University

Liang-Yu Xiong

Zhangshu Municipal People's Hospital

\section{Zu-Tai Huang}

Jiangxi Provincial People's Hospital Affiliated to Nanchang University

\section{Xin Xiao}

Medical college of Nanchang University

\section{Su-Li Zhang}

Wujin Hospital Affiliated to Jiangsu University

\section{Wen-Yan Ni}

Wujin Hospital Affiliated to Jiangsu University

\section{Liang Deng ( $\nabla$ dengliang001137@163.com )}

Jiangxi Provincial People's Hospital Affiliated to Nanchang University https://orcid.org/0000-00027221-9146

\section{Research article}

Keywords: wrist fracture, biomechanical assessment, finite element analysis, wrist protector

Posted Date: May 18th, 2020

DOI: https://doi.org/10.21203/rs.2.24404/v2

License: (c) (1) This work is licensed under a Creative Commons Attribution 4.0 International License. Read Full License 
The authors have withdrawn this preprint from Research Square 